\title{
Study of conjugated polymer blend films by a near field scanning optical microscopy
}

\author{
P. K. Wei ${ }^{a}$, J. H. Hsu ${ }^{a}$, W. S. Fann ${ }^{a, b, *}$ \\ a. Institute of Atomic and Molecular Sciences, Academia Sinica, P.O. Box 23-166, Taipei, 10764, Taiwan \\ b. Department of Physics, National Taiwan University, Taipei, 10764, Taiwan
}

\begin{abstract}
A dual color $(400 \mathrm{~nm}$ and $500 \mathrm{~nm}$ wavelengths) near-field optical microscope (NSOM) was used to study the blend films, made by conjugated polymers, PPV copolymer and PdOPV. These blend films were found to undergo spinodal decomposition with the domain sizes ranging from $10(1-500 \mathrm{~mm}$. The topographic and NSOM images lost correspondence when the degree of decomposition is small. keywords : Conjugated polymers, Near field optical microscopy, phase separation
\end{abstract}

Conjugated polymers, such as poly(p-phenylene-vinylene) (PPV) and its derivatives, are useful materials for light emitting diodes (LEDs) applications. The emission properties can be modified either by chemically changing the side groups of the polymers or physically blending two or more polymers together. The blend polymers have several advantages: (1) Broad emission spectrum can be obtained by blending polymers with different energy gaps[1]. (2) If phase separation occurs in the blend film, the color emitting by the blend polymers can be partly controlled by applied voltages[2]. (3) It can be used as a photodetector [3]. However, in the blend films, the topography and the composition suffer non-uniformities due to the phase separation. The roughness of the surface and the degree of decomposition strongly affect the performance of the LED. Hence, mapping the topography and composition variations is important for improving the polymer blend LEDs. However, the domain size of the blend film is often too small to observe by conventional optical microscopy. The near field scanning optical microscopy (NSOM)[4] has the unique ability of simultaneously mapping optical properties and topography with nanometer scale. Hence, it can be a powerfil tool to investigate the surface properties of blend films. [5]

The blend films studied here are comprising of PPV copolymer and Poly(dioctyloxy phenylene vinylene) (PdOPV) with various composition ratios. PPV copolymer contains repeating units of finite conjugated chain and non-conjugated chain segments. PPV copolymer and PdOPV were dissolved in chlorofom and then mixed at various ratios. To prepare thin films, the solutions were spin-cast on a cover glass. The polymer film has a thickness about $100 \mathrm{~nm}$. Fig. I shows the absorption spectra with different composition ratios $(x=1$ for pure PPV copolymer and $x=0$ for pure PdOPV). It can be seen that PPV copolymer absorbs the UV light and PdOPV, the green light. The absorption increases in the UV range as $x$ increases. On the contrary, the absorption in green light decreases as $\mathrm{x}$ increases. Hence a NSOM operated at two different wavelengths in these two regions can distinguish the concentration variations from the topographic roughness.

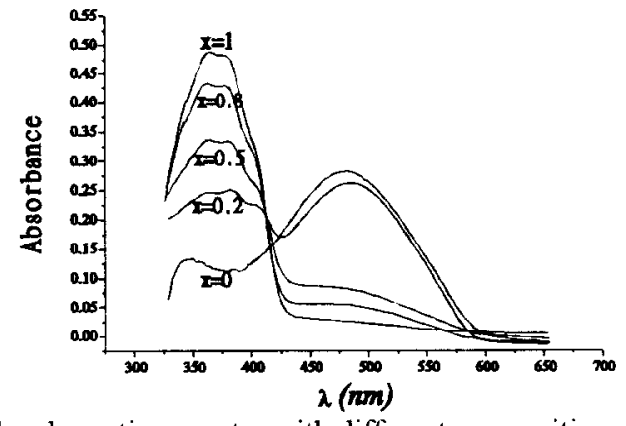

Fig. 1 the absorption spectra with different composition ratios

Fig. 2 shows the photo-luminance spectra of the blend films with different composition ratios. The excited wavelength was $375 \mathrm{~nm}$, except for pure PdOPV which was excited at $514 \mathrm{~nm}$. From Fig. 2, it can be seen that PPV copolymer emits blue light and PdOPV emits orange light. Compared with the absorption spectra shown in Fig. 1, there is a substantial overlap between the emission of PPV copolymer and the absorption of PdOPV. This overlap ensures Förster type energy transfer from PVV copolymer to PdOPV. Owing to this energy transfer, the excited UV light will cause PdOPV to emit luminance. The PL spectra is found to be broad and almost flat as $x-10.9$. Thus, the blend materials are potential candidates for the white light LED.

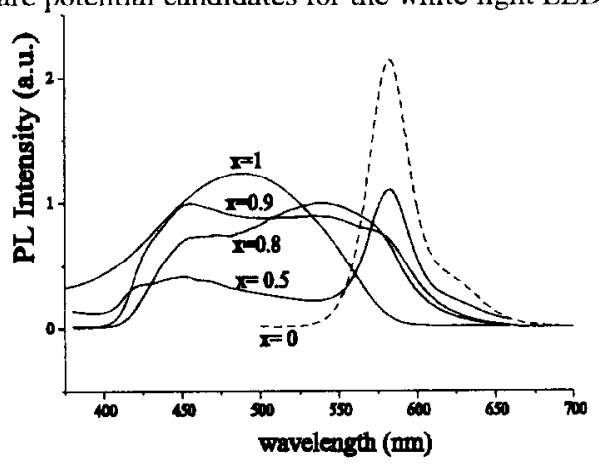

Fig. 2 the PL spectra with different composition ratios

The absorption spectra in Fig. 1 indicate that the absorption can be used to map out the surface properties of the blend films. Hence we use a collection mode NSOM to read the absorption difference. In the near field signal, the operated wavelengths are 
obtained by a 100W $\mathrm{Hg}$ lamp and bandpass filters (10nm bandwidth). Both $400 \mathrm{~nm}$ and $500 \mathrm{~nm}$ wavelengths were selected to investigate the film. The NSOM images at two wavelengths will exhibit opposite contrast, as indicated in Fig. 1, if the optical images are proportional to the concentration variations. The filtered light then focused on the sample surface. A metal-coated tapered fiber probe collected the light signals and sent into a sensitive PMI. For the tip-sample distance regulation, a shear force feedback mechanism with optical detection method was used.

Figs. 3 shows the topographic and NSOM images of the blend films with $x=(0.5$. Fig. 3 (a) is the topography. The surface is relative rough and the mean square roughness is about $200 \AA$ which is $-1 / 5$ of the film thickness. Figs. $3(\mathrm{~b})$ and $3(\mathrm{c})$ are the NSOM images mapped at $500 \mathrm{~nm}$ and $400 \mathrm{~nm}$ wavelengths, respectively. It can be seen that these two images are negatives of each other. A clear phase separation is seen in the NSOM images. The domain size of the blend film ranges from $100 \mathrm{~nm}$ to $500 \mathrm{~nm}$. The difference of the magnitude of the maximum and minimum signal is below $10 \%$. However, if these two polymers are totally separated, the variation should be $-30 \%$. Hence, the decomposition is incomplete. It is spinodal decomposition. The brighter region in Fig. 3(c) should be PdOPV rich region and darker region, PPV copolymer rich region. Furthermore, as compared with Fig. 3(a), the topography is found to have one to one correspondences to the NSOM images. The higher region is the PdOPV rich region.

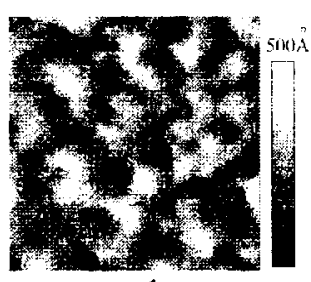

(id) $1 \mu \mathrm{m}$

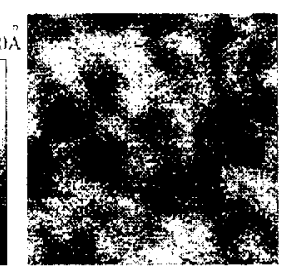

(b)

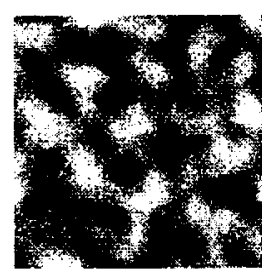

(c)
Figs. 3 the topographic (a) and NSOM images $(b, c)$ of the blend films with $\mathrm{x}=0.5$. Figs. $3(\mathrm{~b})$ and $3(\mathrm{c})$ were mapped at $500 \mathrm{~nm}$ and $40\left(\mathrm{~nm}\right.$ wavelengths, respectively. The scanning area is $2 \times 2 \mu \mathrm{m}^{2}$.

Figs. 4(a) shows the topographic and 4(b) the NSOM images of the blend films with $x=0.9$. The surface becomes smooth and the mean square roughness is about $40 \AA$. Fig. $4(b)$ is the NSOM image mapped at $400 \mathrm{~nm}$. Clear phase separation can also be observed in the NSOM inage. The domain size of this image is similar to that for $\mathrm{x}=0.5$. However, the variation of the detected signal is below $4 \%$. This indicates the degree of decomposition is small. The energy transfer in $\mathrm{x}=0.9$ is also more efficient than that for $x=0.5$. We have also mapped the topography and NSOM images with different composition ratios. It is found that the degree of decomposition is largest when $x \sim 0.5$. As $x$ increases, the degree of decomposition decreases. However, the domain sizes change very little. The average size is $\sim 0.3 \mu \mathrm{m}$. It should be noted that in small degree of decomposition, the topography is not correspondent to the NSOM image as seen in Figs. 4(a) and. 4(b). This means that the NSOM and topography images map two different surface properties.

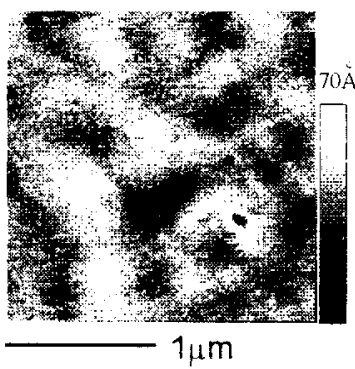

(a)

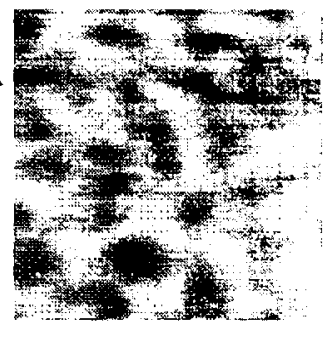

(b)
Figs. 4 the topographic (a) and NSOM (b) images of the blend films with $\mathrm{x}=0.9$. The NSOM image was mapped at $400 \mathrm{~nm}$ wavelength. The area is $2 \times 2 \mu \mathrm{m}^{2}$.

The lack of correspondence of topography and NSOM can be explained by non- iniform distribution of the compositions in the depth direction. In general, the surface roughness, $S(x, y)$, is proportional to the integration of the composition, $C(x, y, z)$, in the depth direction.

$S(x, y) \propto \int^{\prime} c(x, y, z) d z$

where $\mathrm{t}$ is the film thickness. However, the absorption NSOM image, $N(x, y)$, is the convolution between the near field distribution, $I(x, y, z)$, and the composition.

$N(x, y) \propto \iiint C(u, v, z) I(u-x, v-y, z) d u d v d z(2)$

The near field distribution is highly localized at a depth of several hundred $\AA$. Hence the topography represents the summation of the composition in the depth direction, but the NSOM only shows the composition near the surface. If the degree of decomposition is large, a small z-variant composition would not affect the correspondence of the NSOM and topographic images. However, as the composition variation becomes small, a small z-variant distribution would cause the lacking of correspondence between the NSOM and topographic images.

In conclusion, a dual color collection mode NSOM was used to investigate the polymer blend films. It is found that phase separation occurs when PPV copolymer and PdOPV are mixed together. The domain size is about several hundred nanometers and is independent of the composition ratio. The topography and NSOM lost their correspondence when the degree of decomposition is small. The domain size and degree of decomposition depend on the free energy, surface tension, and the viscosity. Further study on the kinetics of the polymer blend is now in progress

\section{References}

[1] M. Berggren, etc., Nature 372, 444 (1994).

[2] G. Magnus, I. Olle, Appl. Phys. Lett. 68, 147 (1996).

[3] J. J. M. Hall, etc., Nature 376, 498 (1995)

[4] E. Betzig, J. K. Trautman, Science 257, 189 (1992)

[5] J. H. Hsu, P. K. Wei, W. S. Fann, K. R. Chuang and S. A Chen, J. Appl Phys 83, 1782, 1998. 